\title{
Orientation and partial disentanglement in individual electrospun fibers: diameter dependence and correlation with mechanical properties
}

\author{
Marie Richard-Lacroix and Christian Pellerin* \\ Département de chimie and Centre for Self-Assembled Chemical Structures \\ Université de Montréal, Montréal, QC, H3C 3J7, Canada
}

* Corresponding author: Tel.: (514) 340-5762; fax: (514) 340-5290;

Emails: c.pellerin@umontreal.ca; marie.richard-lacroix@umontreal.ca 


\section{ABSTRACT}

Electrospun fibers are versatile materials that exhibit unusual and tunable properties when studied at the single fiber level, including an exponential increase in modulus with a decreasing diameter toward the nanoscale. Understanding the detailed molecular organization giving rise to this behavior is a key for reaching the ultimate goal of controlling their properties and realizing their full potential as 1D materials. In particular, molecular orientation and chain disentanglement are thought to play a critical role but their study in individual fibers has proven extremely challenging. Here, we quantify molecular orientation in more than 100 individual fibers of atactic polystyrene (PS) from the micro- to the nanoscale by polarized Raman spectroscopy and we probe for the first time a disentanglement-related conformation in the same spectra. We observe an exponential increase of both parameters when decreasing the fiber diameter below an impressively large onset of $2.5 \mu \mathrm{m}$, much larger than the typical onset values. The orientation quantified for $500 \mathrm{~nm}$ fibers is among the highest values ever reported for PS samples. A clear correlation is found with the previously published diameter dependence of modulus measured in individual PS fibers. Our results also highlight the longitudinal and radial structural heterogeneity of electrospun fibers and suggest separate mechanisms for molecular orientation and disentanglement, which is shown to be mainly situated in the fiber shell. Finally, we combine our observations in a model describing the evolution of chain organization with fiber diameter.

KEYWORDS: Electrospinning; Molecular orientation; Partial disentanglement; Mechanical properties; Raman spectroscopy. 


\section{INTRODUCTION}

Electrospinning is widely used to produce continuous nanofibers through the application of a high voltage on an entangled polymer solution. Electrospun fibers raise an increasing interest because their small diameter, from tens of $\mathrm{nm}$ to a few $\mu \mathrm{m}$, often leads to unusual or improved physical properties as compared to bulk materials. ${ }^{1-3}$ In particular, AFM and nano-tensile testing studies of individual fibers have led to the exciting discovery of a drastic increase in modulus when reducing the fiber diameter below an onset diameter typically around 500-600 nm. ${ }^{4-7}$ This observation was repeated for several semi-crystalline and amorphous polymers and is now considered as a general phenomenon..$^{5-9}$ The incorporation of electrospun nanofibers in electronic and photonic devices has also led to impressive performance improvements as compared to their thin film counterparts. ${ }^{10-19}$ Nevertheless, the controlled production of highly ordered fibers with improved and tunable properties is hampered by our limited comprehension of the structural factors leading to these unusual properties. ${ }^{8}$

The foremost hypothesis to explain the exponential diameter dependence of modulus is a higher molecular orientation in smaller fibers. Modulus and orientation may be directly correlated, in the simplest scenario, but orientation studies on bundles of fibers have led to conflicting results. For instance, Pai et al. have shown a direct orientation/modulus correlation for poly(trimethyl hexamethylene terephthalamide) fibers, ${ }^{20}$ while Arinstein et al. have observed a linear increase of orientation, rather than exponential, for Nylon 6.6 fibers. ${ }^{9}$ Various models have been proposed to describe the chains organization in oriented fibers, including the formation of confined and oriented nanoscale supramolecular structures, ${ }^{4,9}$ of a core-shell fiber morphology in which the oriented chains would be located either in the shell ${ }^{21}$ or in the core ${ }^{22-25}$, and of mechanically coupled oriented surfaces. ${ }^{5,6}$ The heterogeneous distribution of density and 
molecular organization in fiber cross-sections has recently been highlighted, in agreement with the core-shell model, although the results were again contradictory. ${ }^{21-23}$

Adding to the complexity of the system, it has recently been proposed that the polymer chains are less entangled in electrospun fibers than in the bulk polymer due to the extremely high stretching forces acting on the jet during the fiber formation process. ${ }^{22-26}$ The hypothesis of a lower entanglement density was supported by the formation of short fibers and by X-ray phase contrast imaging of the first few $\mathrm{mm}$ of the electrospinning jet. ${ }^{24-26}$ We have recently shown that partial disentanglement can last until fiber collection, for bundles of continuous atactic polystyrene (PS) fibers, ${ }^{27}$ based on the observation of infrared (IR) bands previously associated to a disentanglement-enabled conformation in PS samples prepared by freeze-drying or by spincoating diluted solutions. ${ }^{27-30}$ In contrast to the above hypothesis, and based on the core-shell morphology of the fibers, we suggested that chains in the shell are kinetically frozen in an incompletely re-entangled state due to the fast solvent evaporation at the jet boundaries. Partial disentanglement is known to increase the thermal and electric conductivities of bulk polymers $^{31,32}$ and was suggested to have a large influence on the modulus ${ }^{22}$ and the melting point $^{33-35}$ of electrospun fibers. The disentanglement hypothesis must therefore be incorporated in models relating the unusual properties of electrospun fibers to their molecular-level organization.

The conflicting results and models explaining the chain organization in electrospun fibers originate, at least in part, from our limited ability to characterize the molecular structure of individual nanofibers. Conventional tools such as X-ray diffraction and IR spectroscopy require studying bundles composed of hundreds of aligned fibers, which often show a broad diameter distribution, in order to achieve an appropriate signal-to-noise ratio. The response is thus a 
convolution of the true molecular characteristics of each fiber with the macroscopic organization and diameter distribution in the mat. To date, structural studies of single fibers have been mainly conducted using selected area electron diffraction (SAED) $)^{36-39}$ and scanning near-field optical microscopy (SNOM) ${ }^{22,23}$ Unfortunately, SAED only probes the crystalline phase, limiting the range of polymers and parameters that can investigated, while SNOM is limited to conjugated polymers when used in the visible wavelength range. The experimental complexity of these techniques has also restricted previous studies to a few individual fibers, preventing from drawing a statistical picture of the evolution of structural parameters with fiber diameter. We have recently shown that confocal Raman spectroscopy provides rich molecular information (including quantitative molecular orientation) at the single fiber scale for semi-crystalline and amorphous polymers and that it enables studying larger quantities of single fibers within a reasonable time. ${ }^{40}$

Here, we study the diameter dependence of molecular orientation and partial disentanglement in individual electrospun fibers of atactic PS. This system is ideal since complications associated to crystallization are avoided. By studying more than 100 fibers with diameters ranging from 500 to $5000 \mathrm{~nm}$, we establish a direct correlation between the orientation and the exponential increase in modulus (using published data ${ }^{6}$ for PS single fibers produced in similar conditions) when decreasing fiber diameter. We further confirm that partial disentanglement is located in the shell and that its amplitude follows the same exponential diameter-dependence as orientation and modulus. Based on these results, we propose a schematic model describing the radial distribution of orientation and partial disentanglement as a function of fiber diameter. 


\section{EXPERIMENTAL}

Electrospinning. Atactic polystyrene (Pressure Chemicals) with a weight-average molecular weight of $900 \mathrm{~kg} / \mathrm{mol}$ (PDI of 1.10) and chloroform (Fisher Scientific) were used as received. Fibers were prepared from 10, 12.5 and $15 \% \mathrm{w} / \mathrm{v}$ solutions in a glass syringe equipped with a $0.41 \mathrm{~mm}$ diameter flat-end needle. A flow rate of $0.4-0.8 \mathrm{ml} / \mathrm{h}$ was imposed with a PHD 2000 syringe pump (Harvard Apparatus). A $15 \mathrm{kV}$ positive voltage was applied to the needle tip using

a CZE 1000R high-voltage power supply (Spellman High Voltage Electronics) while a $2 \mathrm{kV}$ negative potential (Power Designs) was imposed on a rotating disk acting as a counter electrode. The distance between the needle tip and the collector was $15 \mathrm{~cm}$. Small quantities of fibers were carefully transferred on $\mathrm{BaF}_{2}$ windows and dried under vacuum for at least $12 \mathrm{~h}$ prior to analysis by Raman spectroscopy.

Raman spectroscopy. Spectra were recorded in the backscattering geometry using the $632.8 \mathrm{~nm}$ He-Ne laser of a LabRam HR800 spectrometer (Horiba Jobin Yvon) coupled with an Olympus BX41 microscope. The confocal hole and the slit were fixed at 100 and $150 \mu \mathrm{m}$, respectively. The protocol for preparing oriented PS films ${ }^{41}$ and the detailed experimental procedures for Raman measurements on electrospun fibers 40 and for quantifying the orientation parameter $\left(\left\langle P_{2}\right\rangle\right)$ values $^{41,42}$ are provided elsewhere. The polarization of the laser and of the Raman scattering, parallel $(\mathrm{Z})$ and perpendicular $(\mathrm{X})$ to the fiber axis, were selected with the help of a half-wave plate and a polarizer, respectively, such that a complete set of two parallel-polarized spectra (ZZ and XX) and two crossed-polarized spectra (ZX and XZ) could be recorded (first and second letters referring to the incident and scattered polarizations, respectively). A scrambler was inserted in the optical path before the 600 grooves $/ \mathrm{mm}$ holographic grating to minimize its 
polarization dependence. The acquisition time for each spectrum was fixed between $10 \mathrm{~s}$ to 2 min, averaged 5 to 10 times.

\section{RESULTS AND DISCUSSION}

Diameter dependence of orientation in individual PS fibers. PS fibers were electrospun from chloroform solutions at three concentrations in order to produce fibers covering a large diameter range from 500 to $5000 \mathrm{~nm}$. Figure 1 shows the four polarized spectra required for quantifying molecular orientation by confocal Raman spectroscopy for two representative PS fibers with diameters of approximately $3 \mu \mathrm{m}$ and $700 \mathrm{~nm}$. The most commonly used band to probe PS orientation is the totally anti-symmetric skeletal vibration of the benzene ring at $620 \mathrm{~cm}^{-1} .43,44$ The signal-to-noise ratio is extremely good although the scattering cross-section of this band is weak and the diameter of the smaller fiber is similar to the laser wavelength. The intensity of the parallel-polarized spectra ( $\mathrm{ZZ}$ and $\mathrm{XX})$ is identical for the $3 \mu \mathrm{m}$ fiber, indicating that it is completely isotropic. In contrast, the intensity of the $\mathrm{XX}$ spectrum is higher than that of the $\mathrm{ZZ}$ spectrum for the $700 \mathrm{~nm}$ fiber, revealing significant chain orientation. The perpendicular orientation of this band $(\mathrm{XX}>\mathrm{ZZ})$ indicates a global orientation of the PS chains along the fiber axis since the Raman tensor associated with this vibrational mode is perpendicular to the main chain. ${ }^{43,44}$ As expected when dealing with samples with uniaxial symmetry (a valid approximation for fibers), the cross-polarized spectra (ZX and XZ) are almost identical in both cases. 


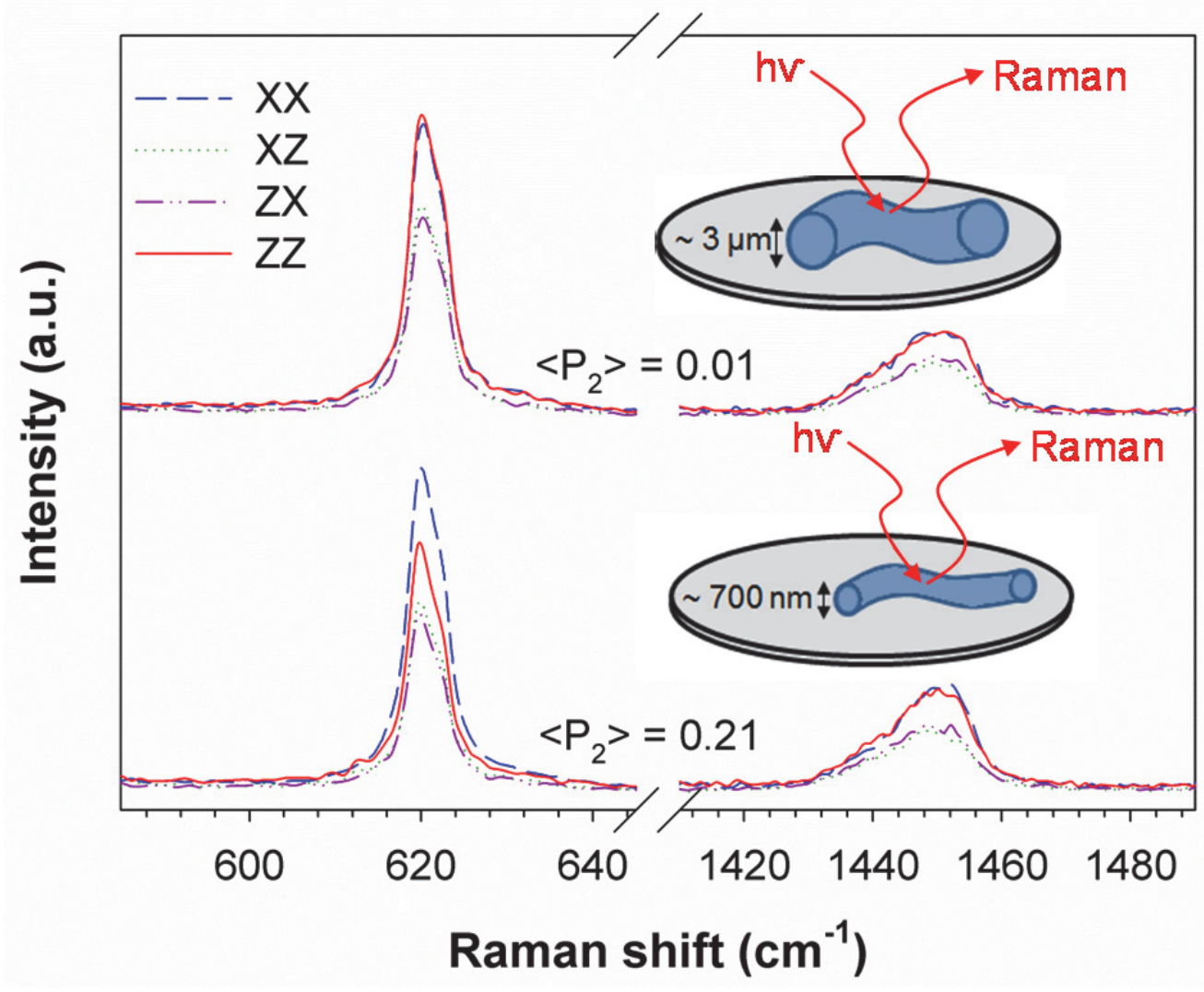

Figure 1. Representative sets of four polarized Raman spectra (required for orientation quantification) recorded for individual PS electrospun fibers with a diameter of $\sim 3 \mu \mathrm{m}$ (top) and $700 \mathrm{~nm}$ (bottom) showing no orientation $\left(\left\langle P_{2}\right\rangle=0.01\right)$ and a high level of orientation $\left(\left\langle P_{2}\right\rangle=\right.$ $0.21)$, respectively.

Quantifying molecular orientation strictly requires calculating the orientation parameter, $\left\langle P_{2}\right\rangle$, which takes limiting values of 0 for a totally isotropic distribution and 1 for a perfect alignment of the polymer chains along the fiber axis. $\left\langle P_{2}\right\rangle$ values of 0.01 and 0.21 are found for the $3 \mu \mathrm{m}$ and $700 \mathrm{~nm}$ fibers, respectively, taking into account the tilt angle of $90^{\circ}$ of the Raman tensor with respect to the chain axis. These values suggest a strong diameter dependence of molecular orientation, as will be demonstrated below. They were quantified using our simplified method 
which eliminates restrictive experimental procedures that often induce errors in the calculation of orientation parameters. ${ }^{42}$ In particular, the most commonly used procedure leads to an overestimation of molecular orientation in the specific case of oriented PS samples. ${ }^{41}$ The accuracy of the $\left\langle P_{2}\right\rangle$ values is reinforced by the intensity equivalence of the $\mathrm{XX}$ and $\mathrm{ZZ}$ spectra for the $1450 \mathrm{~cm}^{-1}$ band for both the isotropic and oriented fibers. This behavior, although not previously reported, was also observed for stretched films covering the full range of orientation for PS samples (data not shown). It strongly supports the absence of any drift during (or between) the acquisition of the 4 polarized spectra and confirms the equivalence of the focus quality for both polarizations. Nevertheless, acquiring 4 polarized spectra on micro- or nanoscale fibers is extremely time consuming considering the numerous experiments that must be rejected based on previously described criteria. ${ }^{40}$ Not respecting these stringent conditions could induce large errors in the $\left\langle P_{2}\right\rangle$ values of individual fibers. To circumvent this issue, a calibration curve enabling the quantification of $\left\langle P_{2}\right\rangle$ using a single polarized Raman spectrum was established using 35 fibers and validated by 7 oriented films (see Figures S1 and S2 in the supporting information).

The molecular orientation of 100 additional PS fibers covering the $500-5000 \mathrm{~nm}$ diameter range was quantified using the calibration procedure. Figure $2 \mathrm{~A}$ shows the evolution of $\left\langle P_{2}\right\rangle$ as a function of diameter, averaged over ranges of $\sim 200 \mathrm{~nm}$. The largest fibers, with diameters from 5000 to approximately $2500 \mathrm{~nm}$, are essentially isotropic with $\left\langle P_{2}\right\rangle=0$ within experimental error. In sharp contrast, reducing the diameter below $2500 \mathrm{~nm}$ clearly leads to an exponential increase of the average $\left\langle P_{2}\right\rangle$ values, as supported by the high correlation coefficient associated to this fit. Some of the smallest fibers (diameter $\sim 500 \mathrm{~nm}$ ) reach unexpectedly high orientation for PS samples with $\left\langle P_{2}\right\rangle$ values as high as 0.3 . Such large values have only been reported for PS 
films cold drawn in the Tg region ${ }^{45}$ and for stretched blends of PS with a miscible polymer, ${ }^{46}$ emphasizing the extremely large stretching forces to which these smallest fibers must have been submitted during their formation. To the best of our knowledge, the only other study of orientation in PS electrospun fibers (using small angle neutron scattering) led to moderate orientation values $\left(\left\langle P_{2}\right\rangle<0.15\right)$, even after considering the alignment of fibers within the studied bundles. ${ }^{47}$

It can be observed in Figure 2A that the standard deviation increases strongly for fibers with a diameter below $2.5 \mu \mathrm{m}$. In fact, Figure S3 reveals that a broad distribution of orientation (with $\left\langle P_{2}\right\rangle$ of 0 vs. 0.21$)$ exists for small fibers with a very similar diameter in spite of a good signal-tonoise ratio and identical XX and ZZ intensities for the orientation-insensitive band at $1450 \mathrm{~cm}^{-1}$. We thus associate this observation to a real distribution of $\left\langle P_{2}\right\rangle$ values rather than to experimental uncertainty. We also observed an analogous broad distribution in a previous study of poly(ethylene terephthalate) electrospun fibers. In that case, the $\left\langle P_{2}\right\rangle$ values were quantitatively correlated with the fraction of trans conformers associated to the formation of a mesophase made possible by the orientation of the chains. ${ }^{40}$ These results highlight the reality of the distribution of structural characteristics for fibers of a given diameter and, thus, the requirement of probing large quantities of fibers for an accurate diameter-orientation correlation to emerge. It should be stressed that the large distribution of $\left\langle P_{2}\right\rangle$ values in Figure $2 \mathrm{~A}$ is highly reminiscent of that observed for mechanical properties measured at the single fiber level, suggesting that they share the same origin in the chaotic nature of jet elongation in the whipping zone during the electrospinning process. ${ }^{9,20}$ In the context of this study, the use of a rotating collector might have induced additional stretching of the fibers and contributed to the extremely large orientation in 
the smallest fibers. Further investigations are necessary to discriminate the relative contributions of the collector and of the fiber formation process.
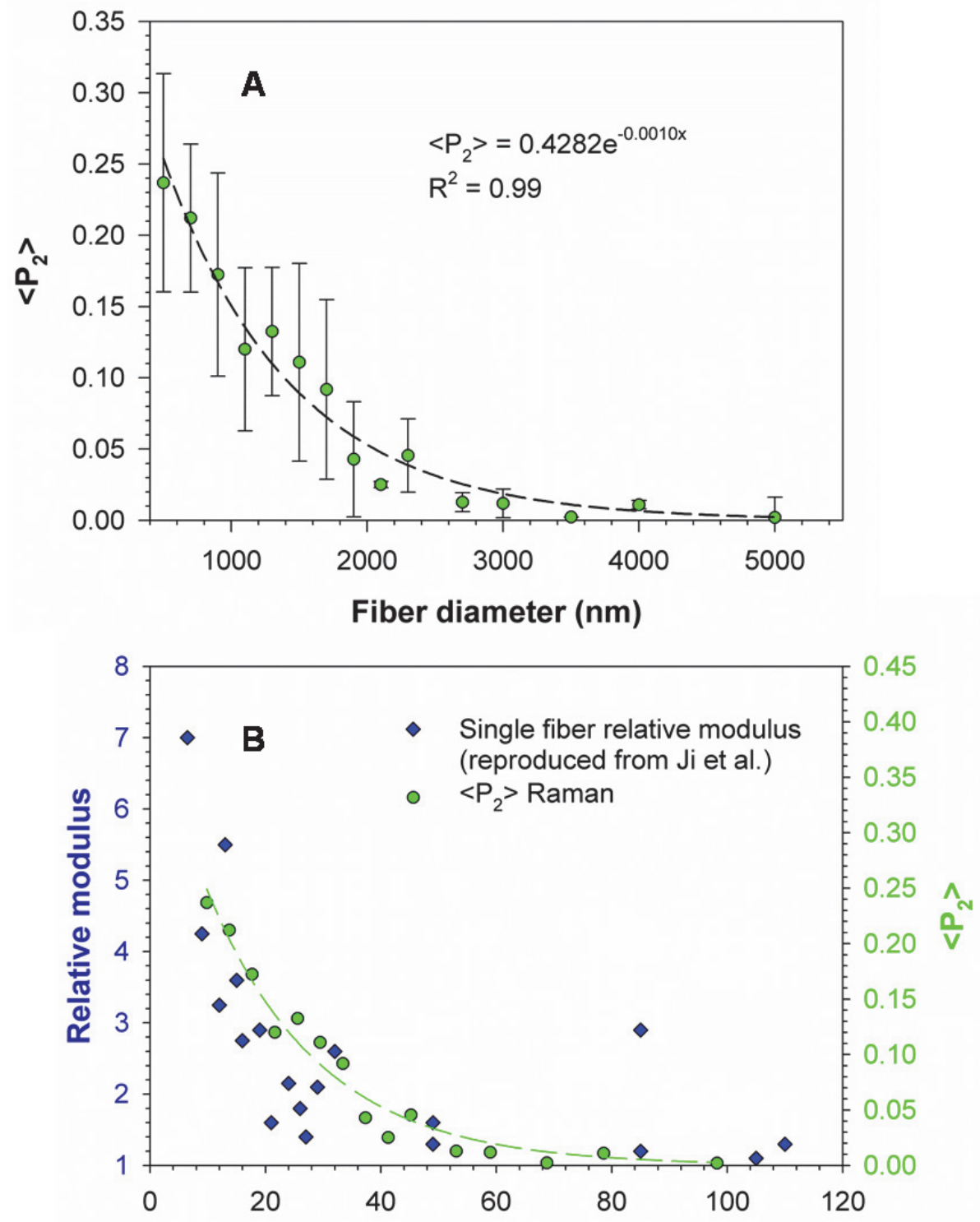

Fiber radius/Rg

Figure 2. A) Exponential dependence of molecular orientation $\left(\left\langle P_{2}\right\rangle\right)$ as a function of the fiber diameter for PS electrospun fibers. The results for $\mathrm{n}=135$ fibers are averaged over $\sim 200 \mathrm{~nm}$ ranges. B) Demonstration of the direct correlation between the diameter dependence of relative modulus and molecular orientation. The modulus values were obtained by Ji et al. ${ }^{6}$ using shear modulation force microscopy at the single fiber level. The fiber radii are normalized by their 
radius of gyration $(\mathrm{Rg})$ to take into account the use of PS samples with different molecular weights.

Interestingly, the onset point for the beginning of the exponential increase in orientation is situated at approximately $2500 \mathrm{~nm}$, much larger than the 500-600 $\mathrm{nm}$ range often reported in studies of the modulus evolution with the fiber diameter. ${ }^{8}$ This impressively large onset diameter can be explained by the high molecular weight of the PS used in this work. Ji et al. have studied the mechanical properties of single fibers of PS (produced in conditions similar to this study) over a large range of molecular weights using AFM-based shear modulation force microscopy and three-point bending techniques. They observed an exponential increase of modulus with a decreasing fiber diameter in all cases and found that, when normalizing the fiber radius with the radius of gyration $(\mathrm{Rg})$ of the chains, the diameter-dependence of the modulus becomes essentially independent of molecular weight. ${ }^{6}$ Their relative moduli (normalized with respect to the bulk value) are reproduced in Figure 2B (blue diamonds) as a function of normalized fiber radius. The orientation values of Figure 2A are also reproduced (green circles) after normalizing the fiber radius with a $\mathrm{Rg}$ of $25.45 \mathrm{~nm}$ for our $900 \mathrm{~kg} / \mathrm{mol} \mathrm{PS}{ }^{48}$ Figure $2 \mathrm{~B}$ clearly shows an extremely strong correlation between the modulus and the molecular orientation. To the best of our knowledge, it is the first time that such direct correlation is established between mechanical properties and molecular orientation measured both at the single fiber scale. Since atactic PS cannot crystallize, these results strongly support the hypothesis that the several-fold increase in modulus observed in the past years for several polymers is mainly due to molecular orientation. This does not exclude, however, that stress-induced crystallization can amplify the phenomenon for crystallizable polymers. In addition, as discussed in the following sections, the molecular orientation and chain organization are not necessarily homogeneous throughout the fiber. 
The fact that the onset for the exponential increase of molecular orientation occurs at a large diameter for a polymer with a high molecular weight $(2.5 \mu \mathrm{m}$ for PS of $900 \mathrm{~kg} / \mathrm{mol})$ offers interesting possibilities in the field of electrospinning. Indeed, it avoids the need of producing fibers with extremely small diameters in order to achieve some of their exceptional properties caused by high orientation and/or to take advantage of their diameter-dependent properties. Based on the results of Figure 2B, the usual 500-600 nm diameter onset would be expected, in first approximation, for a PS with $\mathrm{Mw} \sim 40 \mathrm{~kg} / \mathrm{mol}$, a typical molecular weight for many polymers. The onset could reach values as large as $3.7 \mu \mathrm{m}$ for fibers produced from solutions of PS with $\mathrm{Mw} \sim 2000 \mathrm{~kg} / \mathrm{mol}$. In this mindset, it should be possible to transpose the nanoscale properties of electrospun fibers to the micron scale, when appropriate, for any polymer by simply selecting a sample with a higher molecular weight. This would allow producing larger fibers that are much more convenient to manipulate and to characterize by techniques with a limited spatial resolution while preserving an unusually high orientation and improved diameter-dependent properties.

Partial disentanglement in individual polystyrene fibers. We have recently shown that PS fibers prepared in the same conditions as here present "new" IR bands that indicate the presence of chains adopting an out-of-equilibrium conformation associated with a level of chain entanglement lower than in the bulk. ${ }^{27}$ The same bands were previously reported only for freezedried samples and for ultrathin spin-coated films, ${ }^{28-30}$ samples for which it is well accepted that the chains are kinetically frozen in a partially disentangled state. ${ }^{28,29,49,50}$ Sasaki et al..$^{29}$ concluded that partial disentanglement enables an expanded conformation, much less probable in the bulk, due to the presence of at least one of these bands in the spectrum of crystalline isotactic PS. 


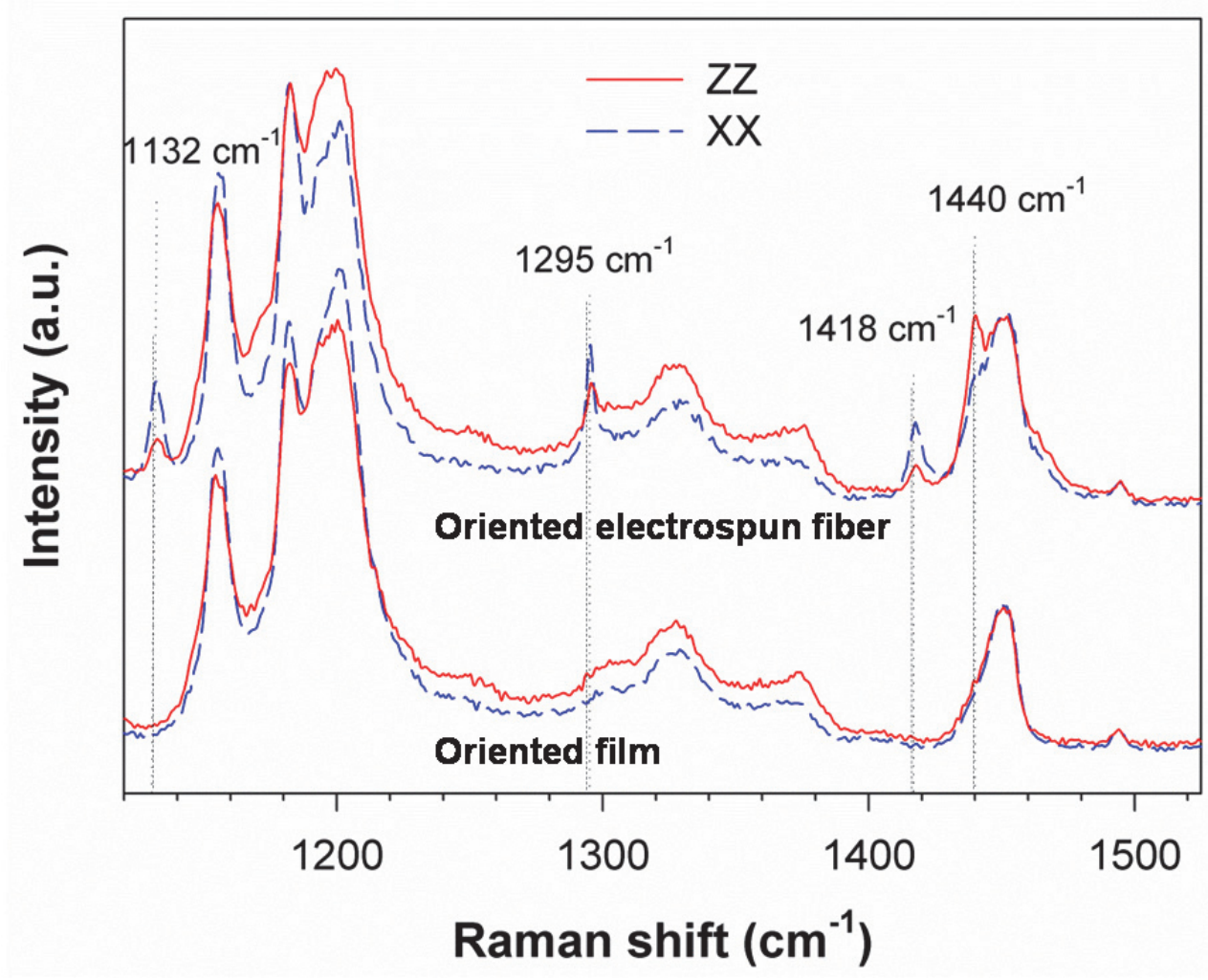

Figure 3. Comparison of the parallel-polarized Raman spectra of an electrospun PS fiber of 500 $\mathrm{nm}\left(\left\langle P_{2}\right\rangle=0.17\right)$ with those of an oriented PS film $\left(\left\langle P_{2}\right\rangle=0.20\right)$. New bands in the fiber spectra are highlighted and are associated to a conformation made possible by partial chain disentanglement.

In a similar way, we observed for the first time unexpected bands in the Raman spectra of some PS fibers. Figure 3 highlights the differences between the $\mathrm{ZZ}$ and $\mathrm{XX}$ spectra of a $500 \mathrm{~nm}$ PS fiber (top) in the $1120-1520 \mathrm{~cm}^{-1}$ spectral range with those of an oriented film (bottom). These samples have similar $\left\langle P_{2}\right\rangle$ values, 0.20 for the film and 0.17 for the fiber. New bands at $1132,1295,1418$ and $1440 \mathrm{~cm}^{-1}$ are clearly discernable in the spectra of the fiber, in addition to a new shoulder around $1230 \mathrm{~cm}^{-1}$ and the possible presence of other bands overlapped with the intense bands around $1200 \mathrm{~cm}^{-1}$. Interestingly, two of these new bands $\left(1295\right.$ and $\left.1440 \mathrm{~cm}^{-1}\right)$ are also present in the Raman spectra of crystalline isotactic PS adopting a 3/1 helix 
conformation. ${ }^{51,52}$ It is noteworthy that chloroform does not share these Raman bands and that no traces of contamination were noticed in the IR spectra of the fibers. The absence of the new bands in the spectra of oriented films confirms that bulk orientation does not lead to this specific conformation. Consequently, we associate these new Raman bands to a conformation made possible by partial chain disentanglement in electrospun fibers.

Our previous IR study suggested that partial disentanglement is related to the formation of a shell, as revealed by a wrinkled fiber morphology in the SEM pictures of Figure S4. When using a highly volatile solvent such as chloroform, a polymer-rich region is created at the edges of the electrospinning jet due to the fast solvent evaporation. ${ }^{25,53}$ This limits the mobility of the polymer chains and hinders their complete re-entanglement. The final level of entanglement should therefore be intermediate between the bulk value and that in the partially entangled semi-diluted initial solution. ${ }^{27}$ The shell is denser than the core when it forms and acts as a wall that severely slows the evaporation of the solvent remaining in the core. Its eventual evaporation induces a buckling that gives rise the wrinkled morphology observed in Figure S4. ${ }^{54,55}$

Confocal Raman spectroscopy enables mapping the longitudinal and radial distribution of the new bands in fibers. The intensity of the $1295 \mathrm{~cm}^{-1}$ band was chosen for this purpose due to its common characteristics with the $1262 \mathrm{~cm}^{-1}$ IR band (used in our previous work) in terms of position and association with crystalline isotactic PS. However, it is not perfectly isolated and appears as a very weak shoulder in the spectra of films. One must eliminate this bulk contribution to compare quantitatively fibers produced in different conditions and/or at different positions on a single fiber. The orientation effects (further discussed below) on its intensity must also be taken into account. For this purpose, we calculated an "isotropic" intensity using the ZZ and XX spectra ${ }^{40}$ and used oriented PS films as a baseline comparison. This led to the evaluation 
of the "EID" value, the Excess of isotropic Intensity associated to a conformation made possible by Disentanglement. The procedure is described in more details in the supporting information. EID values higher than $20 \%$ will be associated to partial disentanglement (for comparison, the largest value obtained for a highly oriented film was $15 \%$ ).

Figure 4A shows the optical microscopy image of a PS fiber, for which the core-shell morphology is clearly discernible, along with the EID values quantified at three radial locations using the $\mathrm{ZZ}$ and $\mathrm{XX}$ spectra shown in Figure 4B. The much higher EID on the edges, as compared to the center of the fiber, supports the hypothesis that partial disentanglement mainly appears in the shell. The spatial resolution of Raman spectroscopy $(\sim 1 \mu \mathrm{m}$, represented by the colored circles) is much larger than the expected thickness of the shell ${ }^{21,22}$ such that the shell cannot be fully isolated from the core and the EID values result from their combined contributions. They nevertheless clearly expose the heterogeneity of the radial distribution and the primary localization of the partially disentangled chains.

According to the core-shell model, large beads should be composed of chains in their bulk-like state (i.e. fully entangled and isotropic) since the evaporation of the massive quantity of solvent entrapped in this enormous core, after the shell solidification, should induce the relaxation of molecular orientation and the loss of any conformation associated to partial disentanglement. Indeed, all large beads analyzed (not shown) were found to be isotropic and to possess an EID similar to that of films. However, Figure 4C shows that the case of elongated beads is more complex. The EID values at the left edge of this bead are comparable to those at the edges of the fiber shown in Figure 4A, but those on the right edge of the bead are much smaller. Since the core of this specific bead is thicker close to its right edge, it occupies a larger fraction of the sampled volume and dominates the Raman signal, leading to low EID values. 


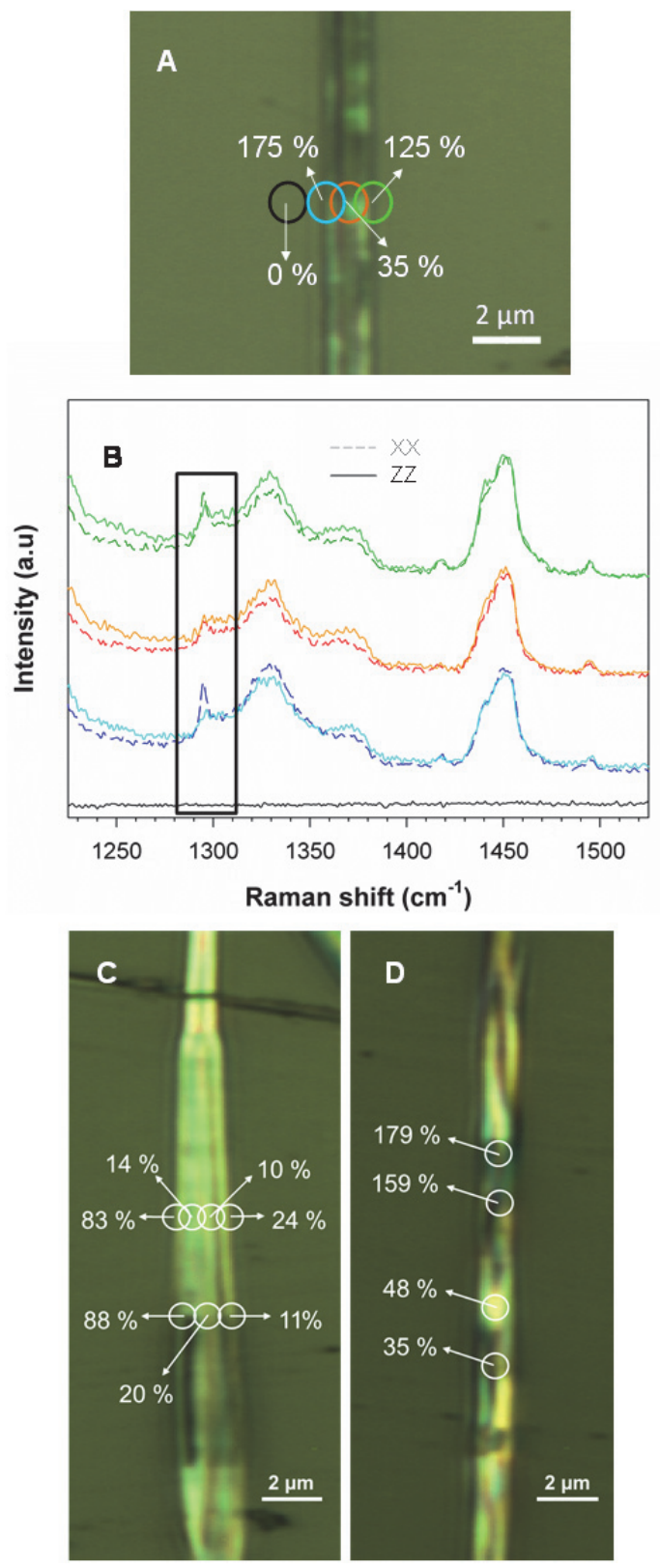

Figure 4. A) Radial Raman mapping of the Excess of isotropic Intensity of the $1295 \mathrm{~cm}^{-1}$ band associated to partial Disentanglement of the chains (EID) for a fiber showing a wrinkled morphology on the micrometer scale. B) Parallel-polarized Raman spectra recorded at the similarly-colored mapping positions shown in panel A. C) Raman mapping of the EID of an 
elongated bead showing a core-shell morphology. D) Longitudinal Raman mapping of a fiber showing a wrinkled morphology.

Most fibers (and beads) do not show a core-shell morphology as apparent as those in Figure 4A and C (i.e. with a thick core surrounded by a collapsed shell) when observed by optical microscopy. Most fibers, such as the one shown in Figure 4D, rather exhibit a wrinkled morphology which is also associated with a core-shell organization in the fiber. In such cases, the EID values are highly heterogeneous along the fiber axis: some sections appear to be dominated by the shell (EID $>100 \%$ ) while others show core-like values, although the fiber does not show any clear diameter variation. This is analogous to the case of molecular orientation for which a large distribution of $\left\langle P_{2}\right\rangle$ values was observed for fibers of a similar diameter.

The EID values were quantified for 135 fibers and are plotted as a function of diameter in Figure 5. For fibers larger than the laser spot (or with the type of morphology shown in Figure $4 \mathrm{~A}$ ), the measurements were made in the center of the fiber. Figure 5A reveals an exponential increase of the EID values with a decreasing fiber diameter which is extremely similar to the diameter-dependence of orientation (reproduced as green circles). The average EID values remain below $20 \%$ (close to the $15 \%$ bulk value) for fibers larger than $2 \mu \mathrm{m}$ but it increases sharply for smaller fibers. The EID results are more scattered than their orientation counterpart, especially for the smallest diameters. Based on the results of Figure 4D, which show a large EID distribution for a given diameter, we believe that this scatter exposes a real distribution of the presence of the conformation made possible by a lower level of entanglement in fibers. However, the results are affected by a much larger uncertainty caused by the low intensity of the $1295 \mathrm{~cm}^{-1}$ band. 

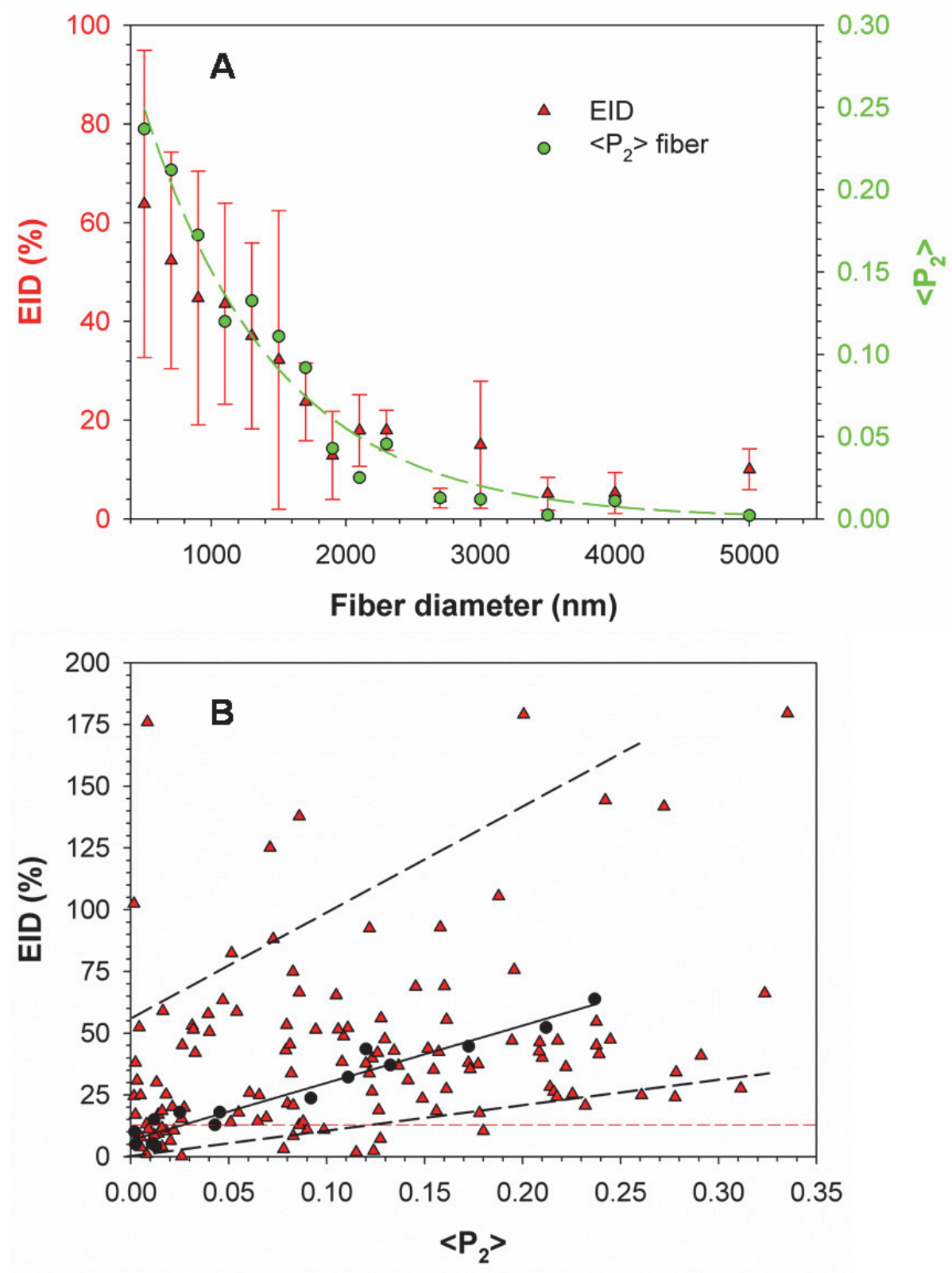

Figure 5. A) Diameter dependence of the Excess of isotropic Intensity of the $1295 \mathrm{~cm}^{-1}$ band associated to partial Disentanglement of the chains (EID) (red triangles). The orientation results are reproduced (green circles) to highlight the similarity of their diameter dependence. B) Orientation dependence of the EID values determined for 135 individual fibers (red triangles). The black circles and plain black line show the linear relationship between the average values of EID and $\left\langle P_{2}\right\rangle$ while the dashed black lines approximately delimit the range of the distribution. 
The dashed red line indicates the largest EID value observed in oriented films and represents a bulk-like state of entanglement.

The results of Figure 5A appear to suggest a direct orientation / disentanglement correlation due to the close similarity of their diameter dependence. Indeed, Figure 5B demonstrates that the average EID values increase approximately linearly as a function of $\left\langle P_{2}\right\rangle$ (black circles and plain black line). However, Figure 5B also shows as red triangles the individual EID / $\left\langle P_{2}\right\rangle$ couples for each of the 135 fibers analyzed, along with additional results obtained when mapping the edges of some fibers (such as in Figure 4). A general increase of EID with orientation can still be observed but it is clear that the spread of the values is extremely large, for any given $\left\langle P_{2}\right\rangle$. In some cases, a high level of orientation is associated with a fairly low amplitude of the disentanglement phenomenon. The opposite is also true, some fibers showing a large EID in spite of a moderate $\left\langle P_{2}\right\rangle$ value. Accordingly, although they share an average diameter dependence that is remarkably similar, orientation and disentanglement are therefore only indirectly correlated. As a consequence, Figure 5B provides key information on the organization of the system by revealing the absence of a causal relationship between these two parameters.

These results are in partial disagreement with the model proposed by Zussman et al. according to which chain orientation and partial disentanglement occur in the core of the fiber due to the large stretching forces acting on the jet. ${ }^{23-26,33-35}$ As a consequence, disentanglement and orientation should be directly correlated because the first occurs as a consequence of the second. In this model, the rapid solvent evaporation leading to the complete solidification of the shell would restrict the occurrence of both phenomena. ${ }^{23-26,33-35}$ This does not correspond to our observations for PS fibers, for which a lower apparent entanglement density is rather found in the shell due to the rapid solvent evaporation that would prevent complete re-entanglement. The 
different origins for stress-induced orientation and for solvent evaporation-induced incomplete re-entanglement would therefore enable the decoupling of the orientation and EID values observed in Figure 5B. Our results do not exclude that stretching-induced disentanglement could also take place and lead to an increase of the amplitude of the phenomenon in the shell while simultaneously inducing it in the core. However, they strongly suggest that this mechanism is not the main driving force for the lower level of chain entanglement in these fibers.

Another important information on the chain organization in PS fibers is provided by the anisotropy of the Raman bands associated to a lower degree of entanglement. So far, we have only considered the $\left\langle P_{2}\right\rangle$ values quantified using the $620 \mathrm{~cm}^{-1}$ band representing the overall state of molecular orientation in the fibers. However, Figures 3 and 4B clearly show that the intensity in the XX spectrum is significantly and systematically higher than in the ZZ spectrum for three of the four new bands (the opposite is observed for the $1440 \mathrm{~cm}^{-1}$ band). This implies that the chains adopting this conformation are highly oriented, most likely along the fiber axis.

Schematic representation of chains organization in PS fibers. Our aim is now to draw an overall picture of the most probable organization of the chains consistent with the diameter dependence observed here for orientation and partial disentanglement and reported by Ji et al. ${ }^{6}$ for the mechanical properties. For this purpose, four main conclusions must be taken into consideration: 1) from Figure 2A, the global molecular orientation increases exponentially when decreasing the fiber diameter, following extremely well the trend reported for the modulus ${ }^{6} ; 2$ ) from the SEM pictures, it can be concluded that the fibers possess a core-shell morphology; 3) from Figure 5A, the average volume fraction of chains showing the conformation enabled by partial disentanglement (the EID values) also increases exponentially with diameter reduction; 
and 4) from Figure 4, these partially disentangled chains are mainly found in the shell and they are systematically highly oriented whenever observed. Taken together, these factors lead to the schematic representation in Scheme 1 of core-shell fibers larger (top), close to (middle) and smaller (bottom) than the onset diameter of $\sim 2.5 \mu \mathrm{m}$.

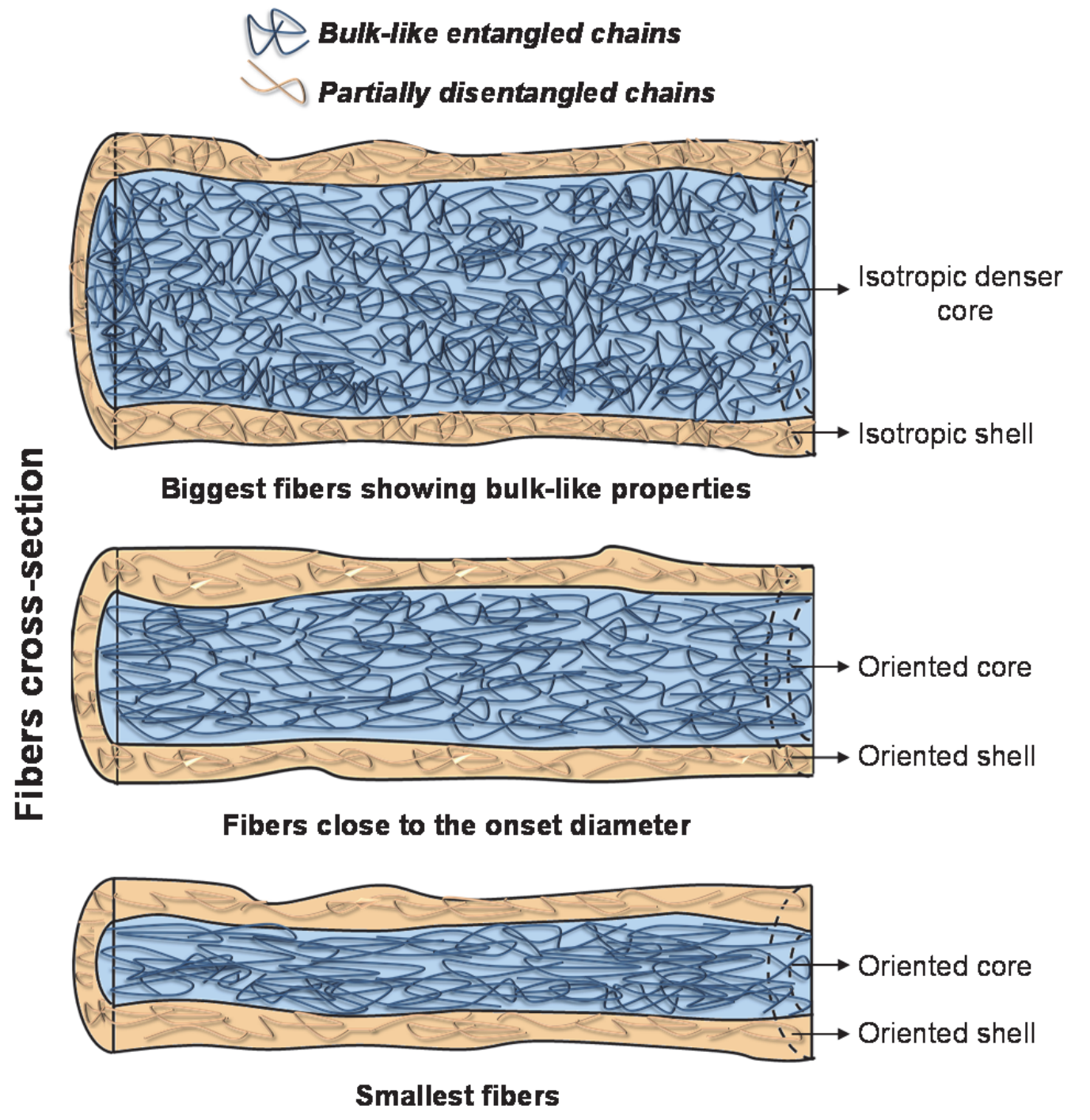

Scheme 1. Schematic representation of the chain organization in PS electrospun fibers showing a core-shell morphology with a constant shell thickness. Fibers are composed of bulk-like entangled chains in the core (blue) and of partially disentangled chains in the shell (orange). For the largest fibers (top), the orientation distribution is isotropic in the core. When reducing the 
fiber diameter close to or below the onset diameter for exponential increase of the modulus, chains in both the shell and the core show a significant level of orientation (middle fiber). Further reducing the fiber diameter toward the nanoscale leads to an increasingly high level of orientation and to an increasing apparent level of disentanglement (EID) due the increasing volume fraction of the shell.

The largest fibers show bulk-like mechanical properties and molecular structure with no orientation and no measurable disentanglement. The core (blue section) is thus composed of isotropic chains with a bulk-like degree of entanglement. Based on the SEM pictures (Figure S4), the shell (orange section) is preserved even for large fibers but, due to its extremely small volume fraction as compared to the core, the $1295 \mathrm{~cm}^{-1}$ band (whose intensity is always small) is not detectable in the Raman spectra. Depending on the fiber diameter, the shell can therefore contain either incompletely or totally re-entangled chains. The orientation of chains in the shell cannot be estimated directly but, knowing that orientation relaxation is a much faster process than chain re-entanglement, ${ }^{56-58}$ we speculate that the slow evaporation of the large amount of solvent trapped in the core of large fibers enables an almost complete orientation relaxation. It is noteworthy that this description of large fibers is also applicable to most beads.

As the fiber diameter is reduced close to and below (middle and bottom of Scheme 1, respectively) the onset value, the situation in the shell becomes more explicit. The spectral markers for the conformation associated to partially disentangled chains are now clearly present and they increase drastically with a decreasing fiber diameter. Since the shell forms at the early stage of the electrospinning process (less than a few $\mathrm{mm}$ from the spinneret), its thickness should be independent of diameter (for fibers produced from solutions at the same concentration). This is in line with the AFM observation by Stachewicz et al. ${ }^{21}$ of a constant average shell thickness 
of $\sim 30 \mathrm{~nm}$ for poly(vinyl alcohol) fibers over a large range of diameters. The increase in EID when reducing the fiber diameter is therefore mainly a simple consequence of the increasing volume fraction of the shell.

It is well established that the polymer density is higher in the shell than in the core, at the moment when it forms, due to the rapid solvent evaporation during the electrospinning process. ${ }^{25,53}$ In contrast, it has been proposed in the literature that the shell is either more ${ }^{21}$ or $\operatorname{less}^{22}$ dense than the core once the fiber has reached its final state and the solvent is completely removed. Although the shell density has never been measured for PS fibers, our results point toward a less dense shell since it would contain polymer chains that are kinetically frozen by rapid solvent evaporation into an incompletely re-entangled state.

The fibers with a diameter below the onset also show an exponentially increasing molecular orientation that is highly correlated with the increasing modulus. It has been suggested in the literature, in the context of a core-shell morphology, that an increasing orientation could be due to 1) a highly oriented shell surrounding an essentially isotropic core (due to slow solvent evaporation enabling orientation relaxation in the core), or 2) an oriented core surrounded by a more or less isotropic shell (because its fast drying would prevent its deformation during the later stages of the electrospinning process). Our results clearly show that the chains in the shell (at least those presenting the Raman conformational markers for incomplete entanglement) are oriented, which is more in line with the first proposition. However, it is very unlikely that the core of the PS fibers remains isotropic. Indeed, the $\left\langle P_{2}\right\rangle$ values quantified for the smallest fibers using the $620 \mathrm{~cm}^{-1}$ band, which reflect the global orientation in the sample, are sometimes close to the maximum value that has ever been reached for PS samples. To mathematically reproduce these results in an oriented shell / isotropic core model, one would need to assume unrealistic 
$\left\langle P_{2}\right\rangle$ values as compared to the highest $\left\langle P_{2}\right\rangle$ values reported for atactic PS stretched films. In addition, the results of Figure 5B revealed that a large $\left\langle P_{2}\right\rangle$ value is sometimes found in spite of a bulk-like EID value, which appears inconsistent with the scenario of an isotropic core.

We thus conclude that the core and the shell both present a significant level of orientation along the fiber axis for fibers below the onset diameter. The core orientation increases for smaller fibers since they must experience a larger overall stretching during their travel towards the collector. The early solidification of the shell implies that all fibers initially entrapped a similar amount of solvent whose presence and eventual evaporation favors orientation relaxation and chain re-entanglement. However, the solvent removal process is sped up for thinner fibers by their stretching-induced diameter reduction, leading to an increasingly larger residual orientation (and to a reduced tendency toward re-entanglement in the shell). According to Zussman et al., ${ }^{22-}$ ${ }^{26}$ stretching could also lead to additional disentanglement either in the shell or in the core of the fiber. Accordingly, reducing the fiber diameter below the onset, as represented at the bottom of Scheme 1, leads to an increase of the volume fraction of the partially disentangled shell and to an exponential increase of orientation in the core (and most likely in the shell). In this scenario, the modulus in the shell should be reduced by the presence of partially disentangled chains and by a lower density, as proposed by Camposeo et al. based on polarized SNOM measurements of poly[2-methoxy-5-(2-ethylhexyloxy)-1,4-phenylenevinylene] fibers. In practice, this effect on the overall modulus is clearly compensated by the increasingly higher orientation of the chains forming the denser core (and possibly by an increasing orientation of the chains in the shell) as the diameter is reduced, leading to the commonly observed exponential increase of modulus.

It is noteworthy that Scheme 1 represents the general tendencies for the averaged $\left\langle P_{2}\right\rangle$ and EID values quantified in this study. As discussed above and shown explicitly in Figure 4, the 
longitudinal and radial distributions of $\left\langle P_{2}\right\rangle$ and EID can be extremely large and they do not necessarily correlate at each point of a fiber. The strength of this model resides in the large number of individual fibers that could be probed by Raman spectroscopy, allowing us to draw statistically meaningful conclusions about the chain organization in amorphous electrospun fibers.

\section{CONCLUSION}

We have established the first orientation / mechanical properties correlation for individual electrospun fibers using atactic polystyrene as a model amorphous system. The results show a direct correlation that supports the controlling role of molecular orientation on the increasing mechanical properties of electrospun fibers when reducing their diameter toward the nanoscale. Probing large quantities of fibers allowed demonstrating the heterogeneity of the distribution for a given diameter while still providing a statistically meaningful diameter correlation for the averaged values. We also observed for the first time Raman bands associated to a conformation made possible by partial disentanglement of the chains. Mapping of the radial distribution of these bands shows that partially disentangled chains are mainly situated in the shell of the fiber, suggesting that they mainly originate from incomplete re-entanglement rather than from stressinduced disentanglement. The average excess intensity of these bands nevertheless follows the same exponential increase as orientation when reducing the diameter due to the increasing volume fraction of the shell. The capability of Raman to simultaneously probe incomplete reentanglement and orientation in a single set of spectra enabled highlighting the absence of direct correlation between these phenomena. A model describing the radial chain organization as a function of fiber diameter is proposed in the context of electrospinning fibers using a volatile 
solvent that enables shell formation, which represents a very common situation. Our aim is now to generalize and refine this model by studying the impact of different solvent parameters and of molecular weight on the chain organization.

\section{ACKNOWLEDGMENT}

This work was supported by a grant and a graduate scholarship (MRL) from the Natural Sciences and Engineering Research Council of Canada (NSERC). We are grateful to F. Byette for his assistance for the SEM imaging.

\section{REFERENCES}

1. Luo, C.J.; Stoyanov, S.D.; Stride, E.; Pelan, E.; Edirisinghe, M. Chem. Soc. Rev. 2012, 41, 4708-4735.

2. Agarwal, S.; Wendorff, J.H.; Greiner, A. Adv. Mater. 2009, 21, 3343-3351.

3. Persano, L.; Camposeo, A.; Tekmen, C.; Pisignano, D. Macromol. Mater. Eng. 2013, 298, 504-520.

4. Arinstein, A. J. Polym. Sci. Part B 2013, 51, 756-763.

5. Ji, Y.; Li, B.; Ge, S.; Sokolov, J.C.; Rafailovich, M.H. Langmuir 2006, 22, 1321-1328.

6. Ji, Y.; Li, C.; Wang, G.; Koo, J.; Ge, S.; Li, B.; Jiang, J.; Herzberg, B.; Klein, T.; Chen, S.; Sokolov, J.C.; Rafailovich, M.H. Europhys. Lett. 2008, 84, 56002.

7. Papkov, D.; Zou, Y.; Andalib, M.N.; Goponenko, A.; Cheng, S.Z.; Dzenis, Y.A. ACS nano 2013, 7, 3324-3331.

8. Richard-Lacroix, M.; Pellerin, C. Macromolecules 2013, 46, 9473-9493.

9. Arinstein, A.; Burman, M.; Gendelman, O.; Zussman, E. Nat. Nanotechnol. 2007, 2, 59-62.

10. Pu, J.; Yan, X.; Jiang, Y.; Chang, C.; Lin, L. Sensor Actuat. A-Phys. 2010, 164, 131-136.

11. Canesi, E.V.; Luzio, A.; Saglio, B.; Bianco, A.; Caironi, M.; Bertarelli, C. ACS Macro Letters 2012, 1, 366-369.

12. Liu, H.; Reccius, C.H.; Craighead, H.G. Appl. Phys. Lett. 2005, 87, -. 
13. Lee, S.W.; Lee, H.J.; Choi, J.H.; Koh, W.G.; Myoung, J.M.; Hur, J.H.; Park, J.J.; Cho, J.H.; Jeong, U. Nano Letters 2009, 10, 347-351.

14. Shin, M.; Song, J.H.; Lim, G.-H.; Lim, B.; Park, J.-J.; Jeong, U. Adv. Mater. 2014, 26, 37063711 .

15. Tu, D.; Pagliara, S.; Camposeo, A.; Persano, L.; Cingolani, R.; Pisignano, D. Nanoscale 2010, 2, 2217-2222.

16. Chen, J.-Y.; Kuo, C.-C.; Lai, C.-S.; Chen, W.-C.; Chen, H.-L. Macromolecules 2011, 44, 2883-2892.

17. Lin, C.J.; Hsu, J.C.; Tsai, J.H.; Kuo, C.C.; Lee, W.Y.; Chen, W.C. Macromol. Chem. Physic 2011, 212, 2452-2458.

18. Ding, B.; Wang, M.; Wang, X.; Yu, J.; Sun, G. Mater. Today 2010, 13, 16-27.

19. Huang, C.; Soenen, S.J.; Rejman, J.; Lucas, B.; Braeckmans, K.; Demeester, J.; De Smedt, S.C. Chem. Soc. Rev. 2011, 40, 2417-2434.

20. Pai, C.-L.; Boyce, M.C.; Rutledge, G.C. Polymer 2011, 52, 2295-2301.

21. Stachewicz, U.; Bailey, R.J.; Wang, W.; Barber, A.H. Polymer 2012, 53, 5132-5137.

22. Camposeo, A.; Greenfeld, I.; Tantussi, F.; Pagliara, S.; Moffa, M.; Fuso, F.; Allegrini, M.; Zussman, E.; Pisignano, D. Nano Lett. 2013, 13, 5056-5062.

23. Camposeo, A.; Greenfeld, I.; Tantussi, F.; Moffa, M.; Fuso, F.; Allegrini, M.; Zussman, E.; Pisignano, D. Macromolecules 2014, 47, 4704-4710.

24. Greenfeld, I.; Arinstein, A.; Fezzaa, K.; Rafailovich, M.H.; Zussman, E. Phys. Rev. E 2011, $84,041806$.

25. Greenfeld, I.; Fezzaa, K.; Rafailovich, M.H.; Zussman, E. Macromolecules 2012, 45, 36163626.

26. Greenfeld, I.; Zussman, E. J. Polym. Sci. Part B 2013, 51, 1377-1391.

27. Richard-Lacroix, M.; Pellerin, C. Macromolecules 2015, 48, 37-42.

28. Sasaki, T.; Tanaka, M.; Takahashi, T. Polymer 1997, 38, 4765-4768.

29. Sasaki, T.; Tanaka, M.; Takahashi, T. Polymer 1998, 39, 3853-3857.

30. Tretinnikov, O.N.; Zbankov, R.G. Macromolecules 2004, 37, 3543-3545.

31. Noriega, R.; Rivnay, J.; Vandewal, K.; Koch, F.P.V.; Stingelin, N.; Smith, P.; Toney, M.F.; Salleo, A. Nat. Mater. 2013, 12, 1038-1044.

32. Shen, S.; Henry, A.; Tong, J.; Zheng, R.; Chen, G. Nat. Nano. 2010, 5, 251-255. 
33. Arinstein, A.; Liu, Y.; Rafailovich, M.; Zussman, E. Europhys. Lett. 2011, 93, 46001.

34. Arinstein, A.; Zussman, E. J. Polym. Sci. Part B 2011, 49, 691-707.

35. Liu, Y.; Chen, S.; Zussman, E.; Korach, C.S.; Zhao, W.; Rafailovich, M. Macromolecules 2011, 44, 4439-4444.

36. Yoshioka, T.; Dersch, R.; Greiner, A.; Tsuji, M.; Schaper, A.K. Macromol. Mater. Eng. 2010, 295, 1082-1089.

37. Yoshioka, T.; Dersch, R.; Tsuji, M.; Schaper, A.K. Polymer 2010, 51, 2383-2389.

38. Ma, X.; Liu, J.; Ni, C.; Martin, D.C.; Chase, D.B.; Rabolt, J.F. ACS Macro Letters 2012, 1, 428-431.

39. Tosaka, M.; Yamaguchi, K.; Tsuji, M. Polymer 2010, 51, 547-553.

40. Richard-Lacroix, M.; Pellerin, C. Macromolecules 2012, 45, 1946-1953.

41. Richard-Lacroix, M.; Pellerin, C. Macromolecules 2013, 46, 5561-5569.

42. Richard-Lacroix, M.; Pellerin, C. Appl. Spectrosc. 2013, 67, 409-419.

43. Jasse, B.; Chao, R.S.; Koenig, J.L. J. Polym. Sci. A1 1978, 16, 2157-2169.

44. Jasse, B.; Koenig, J.L. J. Polym. Sci. A1 1980, 18, 731-738.

45. Jasse, B.; Koenig, J.L. J. Polym. Sci. A1 1979, 17, 799-810.

46. Pellerin, C.; Prud'homm, R.E.; Pézolet, M. Macromolecules 2000, 33, 7009-7015.

47. Mohan, S.D.; Mitchell, G.R.; Davis, F.J. Soft Matter 2011, 7, 4397.

48. Shin, K.; Pu, Y.; Rafailovich, M.H.; Sokolov, J.; Seeck, O.H.; Sinha, S.K.; Tolan, M.; Kolb, R. Macromolecules 2001, 34, 5620-5626.

49. Chen, J.; Xue, G.; Li, Y.; Wang, L.; Tian, G. Macromolecules 2001, 34, 1297-1301.

50. Sun, Q.; Zhou, D.; Wang, X.; Xue, G. Macromolecules 2002, 35, 7089-7092.

51. Cornell, S.W.; Koenig, J.L. J. Appl. Phys. 1968, 39, 4883-4890.

52. Painter, P.C.; Koenig, J.L. J. Polym. Sci. A1 1977, 15, 1885-1903.

53. Guenthner, A.J.; Khombhongse, S.; Liu, W.; Dayal, P.; Reneker, D.H.; Kyu, T. Macromol. Theor. Simul. 2006, 15, 87-93.

54. Arinstein, A.; Avrahami, R.; Zussman, E. J. Phys. D Appl. Phys. 2009, 42, 015507.

55. Pai, C.-L.; Boyce, M.C.; Rutledge, G.C. Macromolecules 2009, 42, 2102-2114.

56. Teng, C.; Gao, Y.; Wang, X.; Jiang, W.; Zhang, C.; Wang, R.; Zhou, D.; Xue, G. Macromolecules 2012, 45, 6648-6651. 
57. Huang, Q.; Alvarez, N.J.; Matsumiya, Y.; Rasmussen, H.K.; Watanabe, H.; Hassager, O. ACS Macro Letters 2013, 2, 741-744.

58. Napolitano, S.; Capponi, S.; Vanroy, B. Eur. Phys. J. E 2013, 36, 1-37. 


\section{For Table of Contents Use Only}

Orientation and partial disentanglement in individual electrospun fibers: diameter dependence and correlation with mechanical properties

Marie Richard-Lacroix and Christian Pellerin*

Table of Contents Graphics

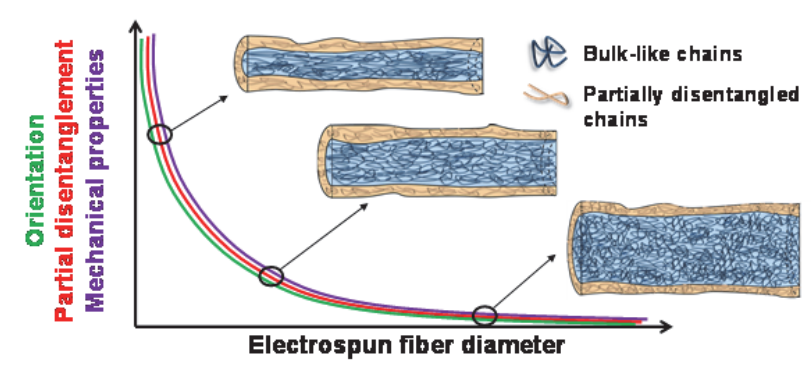

